\title{
Deceleration from Entrainment in the jet of the quasar 1136-135?
}

\author{
F. Tavecchio, L. Maraschi \\ INAF - Osservatorio Astronomico di Brera, via Brera 28, 20121 Milano, Italy \\ R.M. Sambruna \\ NASA/GSFC, Greenbelt, MD 207r1, USA \\ M. Gliozzi \\ George Mason University, Fairfax, VA 22030, USA \\ C. C. Cheung ${ }^{1}$ \\ MIT, Kavli Institute for Astrophysics 6 Space Research, 77 Massachusetts Ave., \\ Cambridge, MA 02139, USA \\ J. F. C. Wardle \\ MS 057, Department of Physics, Brandeis University, Waltham, MA 02454 \\ C. Megan Urry \\ Yale University, Dept. of Astronomy, New Haven, CT 06520, USA
}

\begin{abstract}
Modeling the multiwavelength emission of successive regions in the jet of the quasar PKS 1136-135 we find indication that the jet suffers deceleration near its end on a (deprojected) scale of $\sim 400 \mathrm{kpc}$. Adopting a continuous flow approximation we discuss the possibility that the inferred deceleration from a Lorentz factor $\Gamma=6.5$ to $\Gamma=2.5$ is induced by entrainment of external gas. Some consequences of this scenario are discussed.
\end{abstract}

Subject headings: Galaxies: active - galaxies: jets - X-rays: galaxies - quasars: individual (PKS 1136-135)

\footnotetext{
${ }^{1}$ Jansky Postdoctoral Fellow; National Radio Astronomy Observatory. Now hosted by Kavli Institute for Particle Astrophysics and Cosmology, Stanford University, Stanford, CA 94305
} 


\section{Introduction}

Despite decades of intense efforts, the present knowledge of the physical processes acting behind the phenomenology shown by relativistic jets is still rather poor. Basic questions about matter content, transported power, the role of the magnetic field, the dissipation mechanisms are awaiting answers (e.g. Blandford 2001). Among these problems, one of the most fundamental concerns the speed of the flow. While it is widely accepted that the rapid variability and the extreme compactness displayed by blazars (e.g. Maraschi \& Tavecchio 2005; Sikora \& Madejski 2001) and the observation of VLBI superluminal components (e.g. Kellermann et al. 2004 ) imply relativistic bulk flows (with $\Gamma \sim 10-20$ ) for the innermost portions of the jet $(0.1 \mathrm{pc}<d<100 \mathrm{pc})$, it is not clear whether the large scale jets still have relativistic speeds and how/where deceleration takes place. The present evidence suggests that FRI jets become trans-relativistic quite early, within few kiloparsecs (e.g. Bridle \& Perley 1984), while the situation for FRII jets appears more ambiguous. Mildly relativistic speeds $(\beta<0.95)$ are suggested by studies of high luminosity, lobe dominated sources based on the jet to lobe prominence (Wardle \& Aaron 1997), while the interpretation of multiwavelength observations of extended jets in QSOs points toward highly relativistic speeds $(\Gamma \sim 10)$ even at very large scales $(\sim 100 \mathrm{kpc}$; e.g., Tavecchio et al. 2000, Celotti et al. 2001, Sambruna et al. 2002,2004, Siemiginowska et al. 2002, Marshall et al. 2005).

Deceleration of FRI jets is commonly believed to be the result of entrainment of external gas by the flow (e.g. Komissarov 1994, Bicknell 1994, B94 hereafter). In this framework it is conceivable that FRII jets, characterized by larger powers, are not substantially perturbed by entrainment: the fundamental division between FRI and FRII jets could basically depend on the difference in the mass/energy flux of the two types of jets (Bicknell 1995, Bowman et al. 1996). An important unknown parameter is the efficiency with which the jet can entrain the external gas. Efficient entrainment can be assured by the onset of fully-developed turbulence at transonic speeds (e.g. B94). In this scheme, supersonic flows are thought to be less efficient at collecting material and entrainment could affect only the external regions of the jet, resulting in the formation of a slow layer surrounding a fast, unperturbed "spine" (e.g. de Young 1986). Nevertheless, even low efficiency entrainment could become important if it is maintained over very long scales.

The possibility of "observing" the gradual slowing-down of a jet could in principle provide precious information on the physical processes at work. This approach was successfully developed in great detail for a few FRI jets where the morphology could be well studied thanks to the large angular scale (Laing \& Bridle 2002, 2004). For FR II jets the stronger asymmetry and smaller angular scale has generally prevented comparable studies.

Of particular interest are therefore the cases of FRII jets hosted by intermediate- $z$ 
QSOs in which multiwavelength observations have recently shown that the radio to X-ray flux ratio increases dramatically along the jet. This feature, interpreted in the framework of the synchrotron-IC/CMB emission model, suggests that the jet undergoes deceleration. The possible role played by deceleration was first discussed for the jet of 3C273 (Sambruna et al. 2001). Other cases of jets showing an increasing radio to X-ray flux ratio were presented in the survey discussed in Sambruna et al. (2002) and Sambruna et al. (2004). Georganopoulos \& Kazanas (2004; hereafter GK2004) proposed an analytic framework to describe the effects of deceleration on the physical parameters of the jet on the basis of an adiabatic assumption, remarking that, if the jet decelerates, the magnetic field as well as the particle energy density will increase due to compression causing a large increase of the radio synchrotron emission, while the X-ray flux will decrease because of a reduced beaming of the CMB radiation field. In Sambruna et al. (2005; Paper I in the following) we report the results of deep Chandra and multiwavelenth observations of PKS 1136-135, which allowed us to image in detail the emission along the jet. Modelling the observed multifrequency emission we derived the profiles of the basic physical quantities along the jet, which support the deceleration scenario discussed above. In this paper on the basis of the observational evidence collected for the jet of 1136-135, already presented in Paper I, we discuss in more depth deceleration in terms of physical models and in particular entrainment of external gas.

The plan of the paper is the following: in Sect.2 we derive the profile of the relevant physical quantities, suggesting deceleration of the flow. In Sect.3 we discuss the physical origin of the deceleration. In Sect.4 we discuss in detail the possibility that deceleration is induced by the loading of the jet through entrainment. Finally in Sect.5 we conclude. Throughout this work we use the current "concordance" cosmological parameters: $\mathrm{H}_{0}=$ $71 \mathrm{Km} \mathrm{s}^{-1} \mathrm{Mpc}^{-1}, \Omega_{\Lambda}=0.73, \Omega_{m}=0.27$ (Bennett et al. 2003).

\section{Determining the deceleration profile}

One of the most striking features of the the jet of 1136-135 after knot B is the dramatic increase of its radio luminosity and the associated decay of its X-ray emission (Fig.1). The brightness profiles directly translate into the run of the physical quantities found in Paper I by using the IC/CMB radiative model (Tavecchio et al. 2000; Celotti et al. 2001). Indeed the increasing radio brightness requires an increase of one order of magnitude both of the magnetic field and the density of the radiating electrons (bound together through the equipartition condition). The decrease shown by the X-ray flux, in turn, implies a decrease of the Doppler factor, which reduces the amplification suffered by the target photons of the CMB. Therefore, the observed morphology and the resulting profiles suggest that the special 
behavior displayed by 1136-135 could be related to effects induced by the deceleration of the flow, starting in the region corresponding to knot B-C. Remarkably, the physical parameters inferred for the hot spot ( $\mathrm{F}$ in the prsent work, HS in Paper I) appear to continue the same trend followed by the knots, supporting the emerging view that the hot spots of jets in powerful QSOs can be thought of as slow knots, rather than portions of the jet almost at rest (Georganopoulos \& Kazanas 2003; Tavecchio et al. 2005). We remark that the morphology and the results of the modelling suggest that the deceleration is important only after the region B-C. Before that point the character of the flow seems to be different, with emission clearly concentrated in knots characterized by a similar speed. In this paper we only consider the region after knot $\mathrm{B}$, where deceleration seems to take place.

We review the main features of the model used to fit the data. The emitting region is assumed to be in motion with a bulk Lorentz factor $\Gamma$ at an angle $\theta$ with respect to the line of sight. Since fluxes are extracted within elliptical regions (Paper I), we assume a volume of the emitting region equal to that of the corresponding (deprojected, see below) ellipsoid. The emitting region is homogeneously filled by high-energy electrons, with a power-law energy distribution $N(\gamma)=K \gamma^{-n}$ ( $K$ is the electron normalization) extending from $\gamma_{\min }$ to $\gamma_{\max }$. Electrons emit radiation through the synchrotron and IC/CMB mechanisms. The low-energy limit of the electron distribution $\gamma_{\text {min }}$ is constrained by the optical and the X-ray fluxes. The calculations reported in Paper I have been performed assuming that the observed emission concentrations (knots) mark the presence of discrete moving components of the jet. In the region of the jet under consideration here (after knot B) the flow seems to become more similar to a continuous flow, in which it is difficult to isolate knots (Paper I). It is likely that the flow is complex, showing the characteristics of a continuous flow together with the presence of regions where the dissipation is more efficient, visible as brighter regions in the jet. Based on this evidence, and to be consistent with the scenario that we intend to explore in this paper, we recalculated the physical quantities assuming that, after knot B, the emitting plasma behaves as a continuous flow, moving in a jet whose pattern is at rest with respect to the observer. Practically this implies that the exponent $2+\alpha$ should be used instead of $3+\alpha$ for the Doppler factor to describe the amplification of the radio flux (Lind \& Blandford 1985) and that the observed length is deprojected using the observing angle. The choice of this particular geometry slightly affects the values of the derived quantities, but does not change the evidence of a trend suggestive of deceleration.

With the assumptions above it is possible to derive the required values of the physical quantities by using the observed radio (5 GHZ) and X-ray $(1 \mathrm{keV})$ fluxes. To break the degeneracy between the model parameters (we have three physical quantities the magnetic field, $B$, the electron normalization $K$ and the Doppler factor $\delta$ and two quantities from observations, the radio and the X-ray flux) it is necessary to introduce an additional condition. 
A widely adopted assumption (although, admittedly, not fully motivated) is that particles and magnetic field are in equipartition (e.g. Tavecchio et al. 2004; but see Kataoka and Stawarz 2005). Several evidences indicate the presence of a proton component in jets (e.g. Sikora \& Madejski 2000). However, we assume that protons are not directly coupled to the non-thermal components (relativistic electrons and magnetic field), although they can contribute to the total pressure. We then assume equipartition between relativistic electrons and magnetic field energy densities, $U_{B}=U_{e}$, assuming no contribution from protons. Note that, in any case, the inclusion of protons into the equipartition condition only slightly affects the derived quantities (unless protons strongly dominate the total pressure). It is then possible to find analytical relations providing a unique set of physical parameters characterizing the emission region (e.g. Tavecchio et al. 2000). The appropriate analytic relations are given in Appendix A.

We devoted special attention to the estimate of the uncertainties affecting the final parameters $(B, K$ and $\delta$ ) deriving from the uncertainties associated to the input parameters of the model. To this aim we calculated the ranges covered by $B, K$ and $\delta$ for different values of the input parameters, varying $\gamma_{\min }$ in the range $5-25$, the volume of the emitting region in the range $V-V / 2$, the radio and the X-ray fluxes and the radio spectral slope $\alpha_{r}$ within the corresponding errors (given in Paper I). We find that the total uncertainty is largely dominated by the error on the radio spectral index. We then associate the total range spanned by $B, K$ and $\delta$ for the different input parameters with the total uncertainty.

A more complex discussion involves the viewing angle, $\theta$. It enters as an input parameter in the calculations, through the deprojection of the observed length. At the same time, the angle is constrained by the results of the calculations. Indeed, for a derived Doppler factor $\delta$ (resulting from the modelling), $\theta$ cannot be larger than $1 / \delta$ (see e.g. the discussion in Tavecchio et al. 2004). To fix the angle we then follow an iterative procedure: we fix an angle, we derive the parameters, we check if the maximum Doppler factor required by the knots (including the upper bound deriving from the uncertainties discussed above) satisfies $\theta<1 / \delta_{\max }$. If the condition is not satisfied we reduce the input viewing angle and we repeat the procedure, until we reach the maximum angle allowed by the data, $\theta_{\max }=3.8 \mathrm{deg}$. All the angles $\theta<\theta_{\max }$ are in principle allowed. However, since $\theta_{\max }$ is the most probable angle and the impact of a different choice is quite small and $\theta_{\max }$ is the most probable angle, we use $\theta_{\max }$ in the modelling.

The profiles of $B, K$ and $\Gamma$, derived from the emission model with equipartition, are shown in Fig. 2. The value of the Lorentz factor $\Gamma$ can be derived from $\delta$ once the observing angle is fixed. We recall that each knot is modelled separately from the others: therefore the trends we derive are not by-products of the procedure used, but characteristics of the 
flow, and are basically related to the observed trends of radio and X-ray fluxes along the jet. The evolution of the model parameters with distance are in qualitative agreement with the adiabatic model discussed in GK2004. Unfortunately, the large uncertainties prevent a deeper discussion of this point.

In conclusion our results support the view that the jet of 1136-135 suffers strong deceleration after knot $B$. The Lorentz factor, $\Gamma$ changes, from (with this specific choice of the angle) 6.5 to 2.4 in a projected angular distance of $\sim 4$ arcsec, corresponding to a deprojected linear distance of $\sim 400 \mathrm{kpc}$.

\section{Origin of deceleration}

In the following we consider possible deceleration mechanisms, a question not explicitly addressed in GK2004. First we estimate the possible role of the Compton-Drag effect and then proceed to discuss entrainment.

\subsection{Compton Drag}

A possible way of decelerating a relativistic plasma is through the recoil induced by Compton scattering between the electrons present in the jet and some external radiation field (a process known as Compton Drag effect, e.g. Sikora et al. 1996). This mechanism has been recently proposed as a viable way to decelerate BL Lac jets within the first parsec from the core (Ghisellini, Tavecchio \& Chiaberge 2005) and as a source of IR radiation from interknot regions in large scale jets (Georganopoulos et al. 2005).

In the particular case analysed here the external radiation field is dominated by the CMB (with energy density $U_{\mathrm{CMB}}$ ), while contribution of radio emission from the slow part of the jet (beamed in fast jet frame) is at most comparable to that of the CMB (e.g. Celotti et al. 2001). A key role in determining the efficiency of such a process is played by the composition of the jet: clearly, only when electrons carry a large fraction of the total jet power the process can affect the jet dynamics. Basically, the equation describing the evolution of the bulk Lorentz factor with time can be written as (e.g. Ghisellini et al. 2005):

$$
\dot{\Gamma} \sim \frac{\sigma_{T} c<\gamma^{2}>\Gamma^{2} U_{\mathrm{CMB}}}{m_{e} c^{2}\left(\omega \frac{m_{p}}{m_{e}}+<\gamma>\right)}
$$

where $\omega=n_{p} / n_{e} .\left\langle\gamma^{2}\right\rangle$ and $\langle\gamma\rangle$ depend on the detail of the electron distribution. 
Using typical values derived with the IC/CMB model, $n=2.6, \gamma_{\min }=10, \gamma_{\max }=10^{5}$ (e.g. Sambruna et al. 2004), they can be estimated $\left\langle\gamma^{2}>\sim 10^{4}\right.$ and $\langle\gamma\rangle \sim 25$. We assume the most optimistic case in which electrons do not substantially cool (or are continuously heated) and therefore $<\gamma^{2}>$ is constant. The effects of the deceleration can be estimated through the quantity $r_{\mathrm{dec}}=c \Gamma / \dot{\Gamma}$, yielding the typical distance at which the deceleration takes place. Inserting the numerical values we find $r_{\mathrm{dec}} \sim 100 \mathrm{Mpc}$ for proton-dominated jets $(\omega=1)$, $r_{\text {dec }} \sim 300 \mathrm{kpc}$ for pair-dominated jets $(\omega=0)$ with $\langle\gamma\rangle \sim 10$. We conclude that Compton Drag can be efficient only in the case of a pair-dominated jet. General arguments applied to blazars (although not completely conclusive) seem to point toward a proton component for jets in quasars dominating the dynamics (e.g. Sikora \& Madejski 2000, Ghisellini \& Celotti 2002a, Maraschi \& Tavecchio 2003; see also Uchiyama et al. 2005). We can conclude that an important role of the radiative recoil in determining the inferred deceleration seems unlikely, although not completely ruled-out.

\subsection{Entrainment}

In the following we discuss the possibility that the inferred decline of $\Gamma$ along the jet is caused by the continuous matter loading of the jet through entrainment of external gas entering from the lateral walls of the jet. This mechanism is believed to be responsible for the early deceleration of FR I jets (e.g. B94).

Basically, deceleration through entrainment can be understood to happen through a continuous series of inelastic collisions between the moving plasma and the external gas at rest (e.g., Icke 1991). As a result of the collision, part of the kinetic energy is dissipated and converted into internal energy of the jet, thus increasing the internal pressure. We first derive an approximate formula for the evolution of the Lorentz factor in the simplified case of an entraining confined mass (cylinder). Although drastically simplified, this approach allows to identify the key elements of the entrainment process.

During the motion, the external layer of the cylinder interacts with the external medium, resulting in a continuous increase of the moving mass. Hence a continuous deceleration due to momentum conservation and an increase of internal energy due the dissipation of kinetic energy follow. The dynamics of the entraining mass can be described using the conservation laws for energy and momentum for inelastic collisions. The initial mass is $M_{o}$ with initial Lorentz factor is $\Gamma_{o}$. Assuming that the entrained gas is initially at rest, conservation of energy and momentum yields: 


$$
\begin{aligned}
& \Gamma_{0} M_{0}+m=\Gamma M=\Gamma\left(m+\frac{E}{c^{2}}+M_{0}\right) \\
& \beta_{o} \Gamma_{0} M_{0}=\beta \Gamma M=\beta \Gamma\left(m+\frac{E}{c^{2}}+M_{0}\right)
\end{aligned}
$$

where we have indicated with $m$ the mass of the material entrained by the blob and $E$ is the internal energy accumulated in the collision. Eqs.(2)-(3) give the Lorentz factor $\Gamma$ as a function of the rest-mass $m$ of the collected material:

$$
\Gamma=\frac{a+\Gamma_{o}}{\left(a^{2}+2 a \Gamma_{o}+1\right)^{1 / 2}}
$$

where $a=m / M_{o}$. The Lorentz factor reached depends only on the ratio between the total entrained and initial mass of the blob. For $m / M_{o}<<1 / \Gamma_{o}$ the Lorentz factor remains almost constant, while for $m / M_{o}>>1 / \Gamma_{o} \Gamma$ tends to 1 . Thus the critical scale for deceleration is reached when the collected mass is of order $m_{\text {crit }} \sim M_{o} / \Gamma_{o}$. To halve the initial bulk Lorentz factor $\Gamma_{o}$ the collected mass must be $m=1.5 M_{0} / \Gamma_{o}$. In the limit of the inelastic assumption the collected mass needed to slow down a highly relativistic mass is smaller for higher $\Gamma_{o}$.

In the following we apply the rigorous hydrodynamical treatment developed by B94 to describe the deceleration of the jet of 1136-135 in order to discuss the plausibility of the entrainment mechanism for this particular case. Laing \& Bridle (2002) used the same approach to model the dynamics of the jet of the FRI galaxy 3C31. Our analysis will be not as detailed, due to the more limited information allowed by the larger distance and smaller angular scale. The treatment assumes that the system is adiabatic, the dissipated kinetic energy being stored in the plasma internal pressure. This condition is satisfied in the case under study, for which it is known that the radiative efficiency is quite low (e.g. Tavecchio et al. 2000; Sambruna et al. 2002).

We use momentum and energy conservation in the form given by B94 to follow the evolution of the decelerating flow. An important parameter is $\chi=\rho c^{2} /(\epsilon+p)$, representing the ratio between the rest mass density of the particles within the jet and the sum of internal energy density and pressure (enthalpy). Basically this parameter measures the ratio between the rest-mass energy and the (random) kinetic energy of the particles of the jet, as measured in the comoving frame of the flowing plasma. Values of $\chi>1$ indicate cases for which the average kinetic energy of the particles is less than the rest mass-energy, i.e. a non relativistic plasma. On the contrary values of $\chi<1$ characterize a relativistic plasma. Note that, based on the modelling of the observed emission, we can directly probe only the non-thermal component of the plasma (relativistic electrons and magnetic field). However, 
the plasma can also contain non relativistic or mildly relativistic electrons and protons (the "thermal" component), contributing to (and possibly dominating) the mass density and the total pressure. Defining the initial internal pressure of the jet $p_{0}$, the initial Lorentz factor $\Gamma_{0}$, the initial cross sectional area of the jet $A_{0}$, the initial $\chi_{0}$ parameter and the corresponding downstream parameters, the coupled expressions for momentum and energy conservation read:

$$
(1+\chi) \Gamma^{2} \beta^{2}+\frac{1}{4}=\left[\left(1+\chi_{0}\right) \Gamma_{0}^{2} \beta_{0}^{2}+\frac{1}{4}\right]\left(\frac{p_{0} A_{0}}{p A}\right)
$$

and:

$$
[(\Gamma-1) \chi+\Gamma] \Gamma \beta=\left[\left(\Gamma_{0}-1\right) \chi_{0}+\Gamma_{0}\right] \Gamma_{0} \beta_{0}\left(\frac{p_{0} A_{0}}{p A}\right)
$$

B94 assumed that the dominant role in determining the pressure is played by relativistic particles for which the relativistic equation of state $p=\epsilon / 3$ can be applied; we adopt the same assumption. As can be verified a posteriori, the assumption is fully consistent with the results only at the end of the deceleration process. However, we verified that even in the unrealistic case of a non-relativistic equation of state there are just small corrections to the results (see also Laing \& Bridle 2002) and the general conclusions of our study do not change. Analogously, for simplicity, in Eq.(5) the terms corresponding to the effects of the external medium (expected to be small) have been dropped.

To solve the equation above we assume that the cross sectional area of the jet, $A$, remains approximatively constant from $\mathrm{C}$ to $\mathrm{F}$. Although this assumption is not fully consistent with our choice to neglect the effects of the external medium, the "cilindrical" approximation seems tolerable since the expansion factor in the considered region is at most a factor of 2 . Moreover the jet could be self-confined by, for instance, a large scale magnetic field.

Once the initial state is defined, the coupled non-linear equations can be solved to provide $p$ and $\chi$ at each value of $\Gamma$, considered as a parameter. Other important derived quantities, such as the comoving density $\rho$ and the entrainment rate, can also be calculated.

\section{Results}

The basic features of the deceleration process are shown in Fig. 3 through the evolution of the parameter $\chi$, the density and the pressure as a function of the Lorentz factor reached by the flow. For this calculation we assume initial conditions corresponding to the parameters found above for knot $\mathrm{C}$ of 1136-135, after which the deceleration is more evident (Fig. 2). 
As discussed above, we do not have any information about the thermal components of the plasma. The mass density has been calculated assuming a composition of 1 cold proton per emitting electron. For the pressure at knot C, we assume two cases. In the first, we assume that the pressure is dominated by the non-thermal components, $p_{o}=p_{e}+p_{B}$ (solid lines). In the second case (dashed lines) we assume a pressure ten times that the non-thermal one, thought to be provided by a thermal component.

To satisfy energy and momentum conservation, each small entrained mass induces a small deceleration of the flow and a small increase of the internal energy (assumed to be shared among all the protons inside the jet). This produces variations of the $\chi$ parameter, as shown in Fig. 3 (top). Assuming a jet initially dominated by cold gas $\chi>>1$, the dissipation induced by the continuous loading implies a decrease of $\chi$, because the pressure increases faster than the density of the jet. When the jet is almost completely stopped $\chi$ is of order unity and starts to increase. Note that the regime considered by Laing \& Bridle (2002) for 3C31 corresponds to low $\Gamma$, corresponding to the region where $\chi$ increases during the deceleration. In our application to a highly relativistic FRII jet, we are considering the regime where $\chi$ is large at the beginning and decreases along the jet.

The profile of the comoving density (normalized to the initial value) is shown in Fig.3 (middle). Also shown in the same figure is the profile one would get in the case of deceleration without entrainment (with the same geometry used for the other calculations): in this case the increase of the density only reflects the effect of deceleration and consequent compression of the flow (following the law $\rho / \rho_{0}=\Gamma_{0} / \Gamma$ expressing the conservation of the number of particles). The difference between this curve and the other curves represents the contribution to the total density of the entrained material accumulated along the path. Comparing the densities with and without entrainment it can be seen from Fig. 3 that the entrained mass (proportional to the density difference) needed to decelerate the jet from $\Gamma_{0} \sim 6.5$ to $\Gamma \sim 3.2$ (halving the initial Lorentz factor) represents a small fraction (about 10\%) of the original rest-mass.

In the bottom panel of Fig.3 we report the pressure of the plasma for different Lorentz factors reached by the flow. We also plot (points) the values of the pressure supported by the non-thermal components only (magnetic field and non-thermal electrons), as estimated from the modelling of the emission. The errorbars along the $x$-axis indicate the uncertainty associated to the Lorentz factor of each region, as reported in Fig.2. Clearly the non-thermal component alone can account for a small fraction (around 10\%) of the pressure required by the conservation laws, even in the case in which the initial pressure is completely non-thermal. Therefore much of the pressure is provided by the energy stored within the invisible thermal component. 
The ability of the entrainment process to decelerate the jet is related to the quantity of matter effectively entrained by the jet along its path, expressed by the entrainment rate. The essential parameter characterizing this quantity is the entrainment velocity. Attributing the "observed" deceleration to entrainment we can derive the required entrainment rate and entrainment velocity in the specific case of 1136-135.

The entrainment rate can be defined starting from the law describing the evolution of the jet mass flux (see Eq. 9 of B94):

$$
\Gamma_{2} \rho_{2} \beta_{2} c A_{2}=\Gamma_{1} \rho_{1} \beta_{1} c A_{1}+\int_{1}^{2} \rho_{\text {ext }} v_{\text {ent }} d A
$$

where subscripts 1 and 2 indicate quantities of the jet measured at distances $x_{1}$ and $x_{2}$, $d A \simeq 2 \pi r_{j} d x$ is the jet lateral surface and $v_{\text {ent }}$ is an effective entrainment speed. The (average) entrainment rate is thus defined as:

$$
\frac{\Gamma_{2} \rho_{2} \beta_{2} c A_{2}-\Gamma_{1} \rho_{1} \beta_{1} c A_{1}}{\Delta x_{1,2}}=\frac{1}{\Delta x_{1,2}} \int_{1}^{2} \rho_{\mathrm{ext}} v_{\mathrm{ent}} d A
$$

The average entrainment rate calculated from $\mathrm{C}$ to $\mathrm{F}$ (comparable values can obtained for the intermediate regions C-D, D-E, E-F) is $\sim 2 \times 10^{24} \mathrm{~g} \mathrm{~s}^{-1} \mathrm{kpc}^{-1}$ (the distance has been deprojected assuming $\theta=3.8 \mathrm{deg}$; the inferred entrainment rate would scale as $\propto \theta$ ). For comparison, this value is about an order of magnitude larger than the level required for the deceleration of the jet of 3C31 (Laing \& Bridle 2002). In the latter case the collecting surface is smaller and the density much higher. Further assuming that the external density and the entrainment velocity do not change dramatically from $\mathrm{C}$ to $\mathrm{F}$ and specifying the external density, it is possible to derive the entrainment speed $v_{\text {ent }}$. Assuming that in the region from $\mathrm{C}$ to $\mathrm{F}$ the external gas has a density $n_{\text {ext }}=10^{-5} \mathrm{~cm}^{-3}$ (see below) and that the jet in the region considered here has a radius of $r=10^{23} \mathrm{~cm}$ we find $v_{\text {ent }}=4 \times 10^{7} \mathrm{~cm} / \mathrm{s}$. The corresponding value for the jet of $3 \mathrm{C} 31$ lies around $v_{\text {ent }}=10^{5}-10^{6} \mathrm{~cm} / \mathrm{s}$. Therefore, the entrainment process in 1136-135 appears to be more efficient than that occurring in the low-power jet of 3C31.

\subsection{The onset of deceleration}

A question naturally arising is whether the parameters required by the entrainment model for the "observed" deceleration in the region C-F are consistent with the propagation of the jet from the nucleus to the large scale. For this scope, we consider the simplest 
hypothesis that entrainment is active with the same efficiency (measured by the parameter $\left.v_{\text {ent }}\right)$ all along the path of the jet and assume a conical, entraining jet ${ }^{1}$.

It is possible to check that the critical "cumulative" entrained mass flux for substantial deceleration in the case of a continuous jet satisfies a relation analogous to that derived above for the case of a blob, $m_{\text {crit }}=M_{o} / \Gamma_{o}$. Deceleration becomes important when the contribution of the cumulative mass entrained by a jet slice along its path is of order $1 / \Gamma$ of the jet slice mass. This condition can be put on an explicit form:

$$
\int_{1}^{2} \rho_{\mathrm{ext}} v_{\mathrm{ent}} d A \sim \frac{F_{M, 1}}{\Gamma_{1}}=\rho_{1} \beta_{1} c A_{1}
$$

In order to estimate the entrained mass flux (Eq.9) we need to specify the distribution of the external gas density, $\rho_{\text {ext }} \simeq n_{\text {ext }} m_{p}$ while $v_{\text {ent }}$ will be assumed to be constant. From the Chandra image we infer the presence of faint diffuse emission surrounding the QSO. The distribution of the gas can be modelled as a King profile with core radius $x_{o}=40 \mathrm{kpc}$ and central density $n_{o}=5 \times 10^{-4} \mathrm{~cm}^{-3}$ (Sambruna et al., in prep). This result is consistent with other X-ray observations which show that, in general, nearby $(z<1)$ QSOs lie in environments typical of small groups (Hardcastle \& Worrall 1999; Crawford \& Fabian 2003). At distances of interest here the density can be well modelled as a power-law:

$$
n(x)=n_{o}\left(\frac{x}{x_{o}}\right)^{-1.5}
$$

with $x_{o}=40 \mathrm{kpc}, n_{o}=3 \times 10^{-4} \mathrm{~cm}^{-3}$. From the profile above, the density in the region from knot $\mathrm{C}$ to $\mathrm{F}$ is expected to be of the order of $n_{\mathrm{ext}} \sim 10^{-5} \mathrm{~cm}^{-3}$, which has been used above to derive the value of $v_{\text {ent }} \sim 10^{8} \mathrm{~cm} / \mathrm{s}$. Assuming that $v_{\text {ent }}$ does not substantially change along the jet, it is thus possible to estimate the total matter collected by the jet from the $\mathrm{kpc}$ scale to the hundreds of kpc scale, where deceleration becomes effective. Assuming a conical jet, the area $d A$ can be expressed as $d A=2 \pi r d x$, with $r=r_{o} x / x_{o}$, where $r_{o}$ is the radius at the initial point $x_{o}$ (typically it is assumed $r_{o} / x_{o} \sim 0.1$ ).

Even if the entrainment efficiency is constant and the density of the external medium is decreasing, the entrained mass flux steadily increases, due to the continuous increase of the jet's surface. Calculating the integral in Eq. (9) we find, as expected, that the typical scale

\footnotetext{
${ }^{1}$ In the previous section the jet radius has been approximated as constant in the region from $\mathrm{C}-\mathrm{F}$. However, in the following we want to study the cumulative effect of entrainment from very small to large scales, and thus the increase of the jet radius with the distance must be taken into account.
} 
at which deceleration is effective is $x_{1} \sim 100 \mathrm{kpc}$. Thus the propagation of the jet up to large distances can be understood. The terminal part of the jet of 1136-135 can be identified as the region where the entrained mass becomes critical causing substantial deceleration. We note however that in the schematic model we have drawn with constant entrainment speed, the deceleration profile is very regular. Clearly there must be additional conditions (e.g. the onset of strong instabilities) which enhance the process at particular sites driving the localized occurrence of deceleration.

\section{Discussion and Conclusions}

The proposed scenario can explain in a plausible way the deceleration inferred for the jet of 1136-135 (and possibly in the other sources showing the same radio-to-X-rays increasing trend, GK2004). A more detailed understanding of the processes at work and the comparison with the observed properties of jets necessarily involves several still poorly-known physical issues.

It is worth remarking that the evidence for deceleration in the jet of 1136-135 is based on the observed run of radio and X-ray fluxes along the jet and on the use of a specific emission model to interpret the multiwavelength observations (for a discussion of alternative emission models see Paper I). In this respect, the conclusion about deceleration is not completely model-independent. Other specific assumptions made in the modelling, such as the equipartion condition, affect (although in a minor way) the conclusion. Moreover, apart from the application of a specific emission model, one has to bear in mind that the result is based on the hypothesis that the emission comes from a not-structured flow. At least part of the increase of the radio flux close to the terminal part of the jet could be due to the contribution from another component (e.g. the plasma backflowing after the compression at the hot-spot).

The approach that we used to study the deceleration is based on a pure hydrodynamical treatment. The contribution of all the components of the flow (protons, electrons, magnetic field) is included in just one parameter, the total pressure. Likely, much of the energy dissipated during the deceleration will be stored in the heated proton component. To follow the evolution of the other components (thermal and non-thermal electrons, magnetic field) we should specify the actual mechanisms coupling each component to the others. The determination of the coupling between protons, magnetic field and electrons, determining the fraction of dissipated energy that goes into the magnetic and non-thermal electron components, is a longstanding problem in astrophysics, clearly beyond the scope of this work. The derived profiles are in agreement with the possibility that the increase of the magnetic field 
and of the density of the relativistic electrons just follows the adiabatic heating induced by the deceleration. However, given the large uncertainties, this evidence is not conclusive. The contribution of non-thermal electrons and magnetic field to the total pressure is small, around $10 \%$. Therefore, a large portion of the dissipated kinetic energy resides in the hot protons and is not radiated away.

Another interesting point is related to the nature of the mechanism able to induce the assumed entrainment. It is widely assumed that turbulence can effectively work only in slow (transonic or subsonic) jets. Apart from fully developed turbulence, it is also possible that entrainment is mediated by viscosity effects induced by small-scale turbulence. It is worth noting that in the calculation we have presented it is supposed that the entire jet is decelerated under the effect of the entrainment. More realistically, these processes will induce a radial structure in the jet, with the development of a sheared flow (e.g. De Young 1996).

In the simple scheme presented here, the jet starts to be decelerated when the amount of collected gas is of the order of $1 / \Gamma$ of the mass transported by the jet. In this framework, jets characterized by different mass fluxes will experience different behaviours. Large mass fluxes will assure that, under the same conditions of external gas density and entrainment rate, the jet will reach its hotspot almost unperturbed. On the other hand, jets with a small mass flux will be decelerated soon. It is tempting to further speculate along these lines, connecting the deceleration of the jet to other properties of the central AGN. To this aim we indicate the jet mass flux as $\dot{M}_{\text {out }}$, and we note that $\dot{M}_{\text {out }}$ is the mass output rate of the central engine, which is probably some fraction of the accretion mass rate $\dot{M}_{\text {out }} \propto \dot{M}_{\text {acc }}$ (e.g. Ghisellini \& Celotti 2002b). From the condition expressed by Eq.(9) and the definitions above we can find that the deceleration length $x_{1}$ is $x_{1} \propto \dot{M}_{\mathrm{acc}} / r_{o}$, (the exact dependence is related to the jet and the external density profile) where we have supposed that all the jet, before the deceleration, have a similar bulk Lorentz factor. If the initial radius of the jet scales as the gravitational radius of the central black hole $r_{o} \propto M_{B H}$, we finally find $x_{1} \propto \dot{m}_{\text {acc }}$, where $\dot{m}_{\text {acc }}$ is the accretion rate in Eddington units. Thus it is possible to identify sources for which the jet is effectively decelerated within short scales with sources with low $\dot{m}_{\text {acc }}$ and viceversa, a situation reminiscent of the FRI/FRII division.

We thank Gabriele Ghisellini for useful discussions and the anonymous referee for constructive comments and criticisms. F.T and L.M acknowledge support from grant COFIN2004-023189-005. 


\section{A. Analytical estimate of the physical parameters}

- Synchrotron EC/CMB emission. Assuming a simple power-law distribution for the emitting electrons:

$$
N(\gamma)=K \gamma^{-n}
$$

the synchrotron luminosity can be written as (e.g. Ghisellini et al. 1985):

$$
L_{s}\left(\nu_{s}\right)=c(\alpha) K B^{1+\alpha} V \delta^{p} \nu_{s}^{-\alpha}
$$

where $V$ is the volume of the emitting region, $\alpha=(n-1 / 2)$ is the spectral index, $c(\alpha)$ is a constant and the luminosity is calculated at the frequency (observer frame) $\nu_{s}$. The exponent $p$ of the Doppler factor depends on the geometry of the flow: $p=3+\alpha$ for a moving "blob", $p=2+\alpha$ for a flow whose pattern is fixed in the observer frame (Lind \& Blandford 1985).

To express the luminosity emitted through the IC/CMB ee start from the the standard relation for the inverse Compton luminosity:

$$
L_{c}(\nu)=\frac{4}{3} \sigma_{T} c V U_{\mathrm{rad}} \delta^{p_{1}} \int_{\gamma_{\min }}^{\gamma_{\max }} \gamma^{2} N(\gamma) \delta\left(\nu-\nu_{c}\right) d \gamma
$$

where $p_{1}=4+2 \alpha$ for a "blob" (Dermer 1995) and $p_{1}=3+2 \alpha$ for a continuous flow. The

above expression assumes that the spectrum of the soft radiation is extremely peaked around the frequency $\nu_{o}$ and then $\gamma$ and $\nu_{c}$ can be related by:

$$
\gamma=\left(\frac{\nu_{c}}{\nu_{o}}\right)^{1 / 2}
$$

Using this expression we can rewrite eq. (A3) as:

$$
L_{c}\left(\nu_{c}\right)=\frac{2}{3} \sigma_{T} c V U_{\mathrm{rad}} K \nu_{o}^{\alpha-1} \nu_{c}^{-\alpha} \delta^{p_{1}}
$$

Using (A2) to eliminate $K$, we obtain:

$$
\delta=B \eta
$$

where the factor $\eta$ is:

$$
\eta=\left[\frac{3}{2} \frac{c(\alpha)}{\sigma_{T} c} \frac{1}{U_{\operatorname{rad}} \nu_{o}^{\alpha-1}} \frac{L_{c}\left(\nu_{c}\right)}{L_{s}\left(\nu_{s}\right)}\left(\frac{\nu_{s}}{\nu_{c}}\right)^{-\alpha}\right]^{1 /(1+\alpha)}
$$




\section{- Equipartition condition.}

If we assume equipartition $\left(U_{B}=U_{e}\right)$ between the magnetic energy density $U_{B}$ and the energy density of relativistic electrons:

$$
U_{e}=m c^{2} \int_{\gamma_{\min }}^{\gamma_{\max }} N(\gamma) \gamma d \gamma .
$$

we obtain the following relation between $\delta$ and $B$ :

$$
\delta=\xi B^{-(3+\alpha) / p}
$$

where $\xi$ is:

$$
\xi=\left[\frac{8 \pi m c^{2} L_{s}\left(\nu_{s}\right) f}{c(\alpha) \nu_{s}^{-\alpha} V}\right]^{1 / p} .
$$

The factor $f$ depends on the details of the electron distribution

$$
f=\frac{1}{n-2}\left(\gamma_{\min }^{2-n}-\gamma_{\max }^{2-n}\right) .
$$

Equating Eq.(A9) and Eq. (A6) we can write the magnetic field as:

$$
B=\left(\frac{\xi}{\eta}\right)^{p /(p+3+\alpha)}
$$

and, inserting in Eq. (A6)

$$
\delta=\eta\left(\frac{\xi}{\eta}\right)^{p /(p+3+\alpha)}
$$

The normalization $K$ of the emitting electrons can be derived rearranging the expression of the synchrotron emission, Eq.(A2).

\section{REFERENCES}

Bennett, C. L., et al. 2003, ApJS, 148, 1 
Bicknell, G. V. 1994, ApJ, 422, 542 (B94)

Bicknell, G. V. 1995, ApJS, 101, 29

Blandford, R. D. 2001, ASP Conf. Ser. 250: Particles and Fields in Radio Galaxies Conference, 250, eds. Laing, R.A \& Blundell, K.M., 487

Bowman, M., Leahy, J. P., \& Komissarov, S. S. 1996, MNRAS, 279, 899

Bridle, A. H., \& Perley, R. A. 1984, ARA\&A, 22, 319

Celotti, A., Ghisellini, G., \& Chiaberge, M. 2001, MNRAS, 321, L1

Crawford, C. S., \& Fabian, A. C. 2003, MNRAS, 339, 1163

Dermer, C. D. 1995, ApJ, 446, L63

De Young, D. S. 1986, ApJ, 307, 62

De Young, D. S. 1996, ASP Conf. Ser. 100: Energy Transport in Radio Galaxies and Quasars, 100, eds. Hardee, P.E., Bridle, A.H., Zensus, J.A., 261

Georganopoulos, M., \& Kazanas, D. 2003, ApJ, 589, L5

Georganopoulos, M., \& Kazanas, D. 2004, ApJ, 604, L81 (GK2004)

Georganopoulos, M., Kazanas, D., Perlman, E., \& Stecker, F. W. 2005, ApJ, 625, 656

Ghisellini, G., Tavecchio, F., \& Chiaberge, M. 2005, A\&A, 432, 401

Ghisellini, G. \& Celotti, A. 2002a, ASP Conf. Ser. 258: Issues in Unification of Active Galactic Nuclei, eds. R. Maiolino, A. Marconi \& N. Nagar (San Francisco: ASP), 273

Ghisellini, G., \& Celotti, A. 2002b, in Blazar Astrophysics with BeppoSAX and Other Observatories, Ed. P. Giommi, E. Massaro, G. Palumbo. ESA-ESRIN, 2002. p. 257

Ghisellini, G., Maraschi, L., \& Treves, A. 1985, A\&A, 146, 204

Hardcastle, M. J., \& Worrall, D. M. 1999, MNRAS, 309, 969

Icke, V. 1991, in Beams and Jets in Astrophysics, Cambridge Astrophysics Series, 232

Kataoka, J., \& Stawarz, L. 2005, ApJ, 622, 797

Kellermann, K. I., et al. 2004, ApJ, 609, 539 
Komissarov, S. S. 1994, MNRAS, 269, 394

Laing, R. A., \& Bridle, A. H. 2004, MNRAS, 348, 1459

Laing, R. A., \& Bridle, A. H. 2002, MNRAS, 336, 1161

Lind, K. R., \& Blandford, R. D. 1985, ApJ, 295, 358

Maraschi, L., \& Tavecchio, F. 2005, AIP Conf. Proc. 745: High Energy Gamma-Ray Astronomy, 745, 129

Maraschi, L., \& Tavecchio, F. 2003, ApJ, 593, 667

Marshall, H. L., et al. 2005, ApJS, 156, 13

Sambruna, R. M., et al., 2005, ApJ, in press (astro-ph/0511459)

Sambruna, R. M., Gambill, J. K., Maraschi, L., Tavecchio, F., Cerutti, R., Cheung, C. C., Urry, C. M., \& Chartas, G. 2004, ApJ, 608, 698

Sambruna, R. M., Maraschi, L., Tavecchio, F., Urry, C. M., Cheung, C. C., Chartas, G., Scarpa, R., \& Gambill, J. K. 2002, ApJ, 571, 206

Sambruna, R. M., Urry, C. M., Tavecchio, F., Maraschi, L., Scarpa, R., Chartas, G., \& Muxlow, T. 2001, ApJ, 549, L161

Siemiginowska, A., Bechtold, J., Aldcroft, T. L., Elvis, M., Harris, D. E., \& Dobrzycki, A. 2002, ApJ, 570, 543

Sikora, M., Sol, H., Begelman, M. C., \& Madejski, G. M. 1996, MNRAS, 280, 781

Sikora, M., \& Madejski, G. 2000, ApJ, 534, 109

Sikora, M., \& Madejski, G. 2001, American Institute of Physics Conference Series, 558, 275

Stawarz, Ł., Sikora, M., Ostrowski, M., \& Begelman, M. C. 2004, ApJ, 608, 95

Tavecchio, F. et al. 2000, ApJ, 544, L23

Tavecchio, F., Maraschi, L., Sambruna, R. M., Urry, C. M., Cheung, C. C., Gambill, J. K., \& Scarpa, R. 2004, ApJ, 614, 64

Tavecchio, F., Cerutti, R., Maraschi, L., Sambruna, R. M., Gambill, J. K., Cheung, C. C., \& Urry, C. M. 2005, ApJ, 630, 721 
Uchiyama , Y., et al. 2005, ApJL, 631, 113

Wardle, J. F. C., \& Aaron, S. E. 1997, MNRAS, 286, 425 


\section{Figure Captions}

Fig. 1.- Profile of the X-ray (1 keV) and radio (4.9 GHz) flux along the jet of PKS 1136-135 (data taken from Sambruna et al. 2005).

Fig. 2.- Profiles of the relevant quantities ( $\Gamma$, top panel, $B$ and $K$, lower panel) for regions $\mathrm{B}-\mathrm{F}$ as estimated from the radiative model.

Fig. 3.- The parameter $\chi$ (top), the comoving density (normalized at the initial value, middle) and the pressure (in units of $10^{-12} \mathrm{dyn} / \mathrm{cm}^{-2}$ ) as a function of the Lorentz factor of the jet, calculated assuming the initial conditions inferred for knot $\mathrm{C}$ of the jet of 1136135. Solid lines refer to the case of an initial pressure equal to that of magnetic field and relativistic electrons, while dashed lines report the results assuming a pressure ten times that the non-thermal one. The dotted line in the density panel visualizes the value of the density in the case of deceleration without entrainment. Points overimposed on the pressure curve indicate the value of the pressure provided by magnetic field and non-thermal electrons derived for the different portions of the flow through the modelling of the emission (Fig.2). 


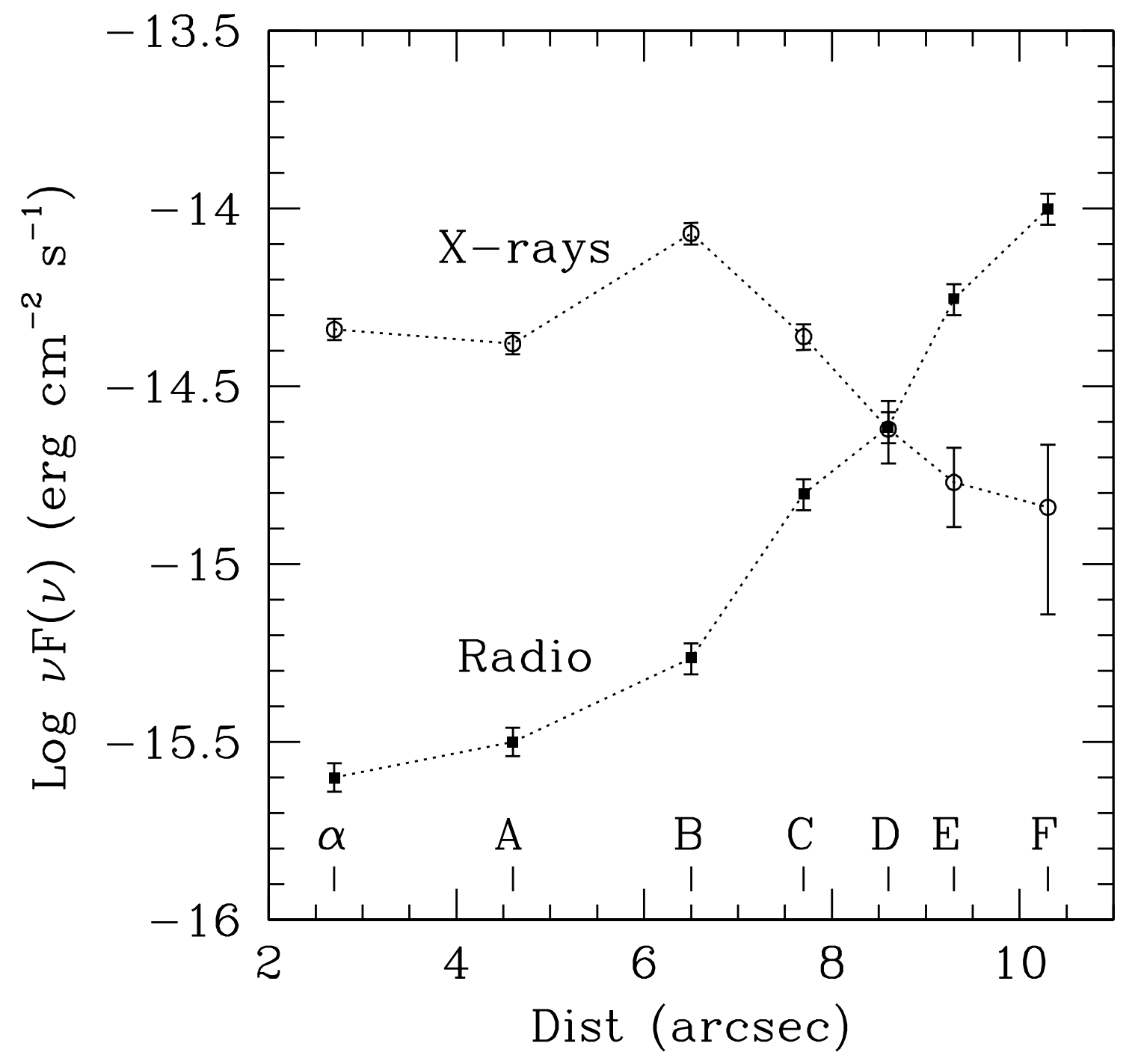




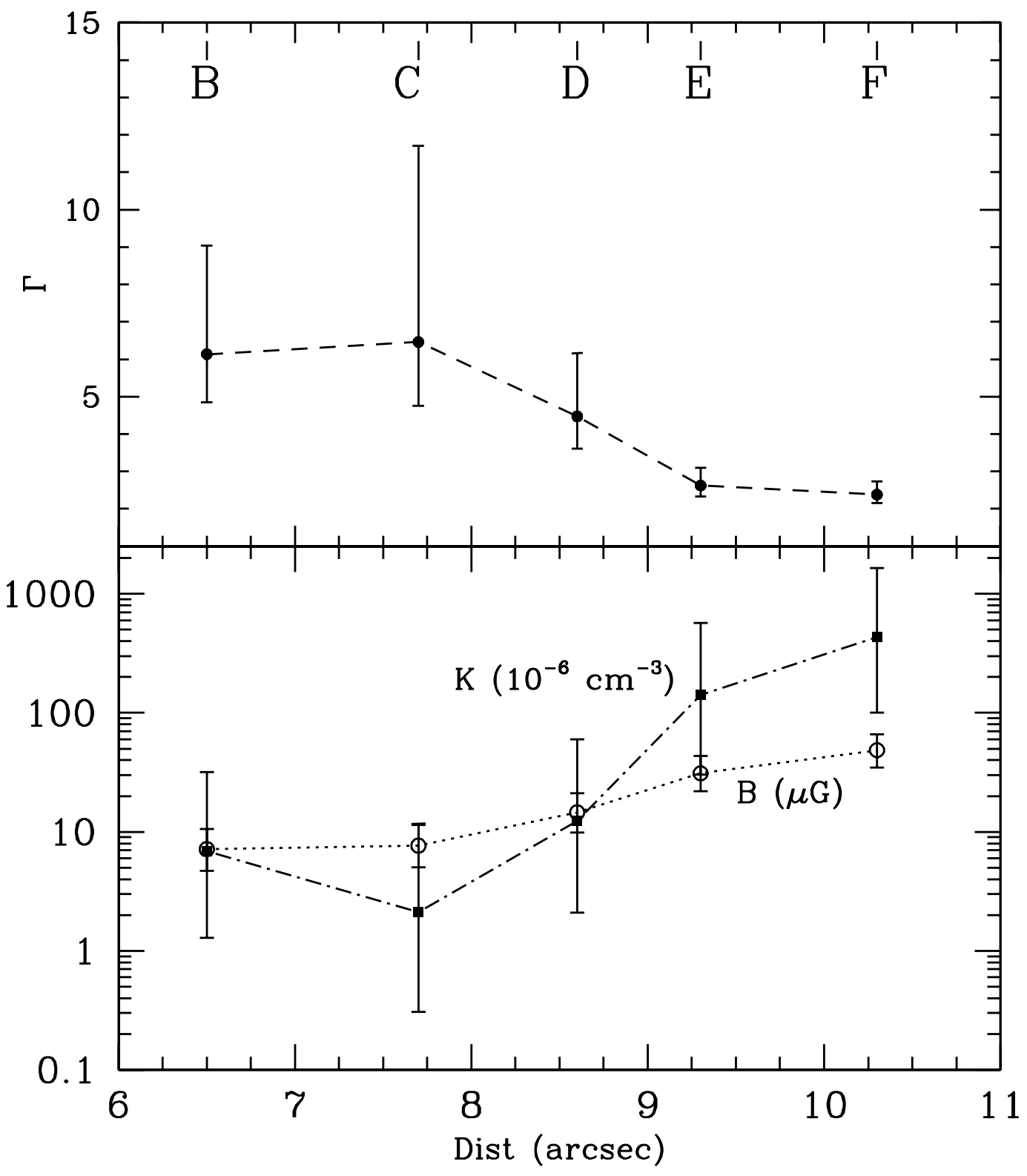




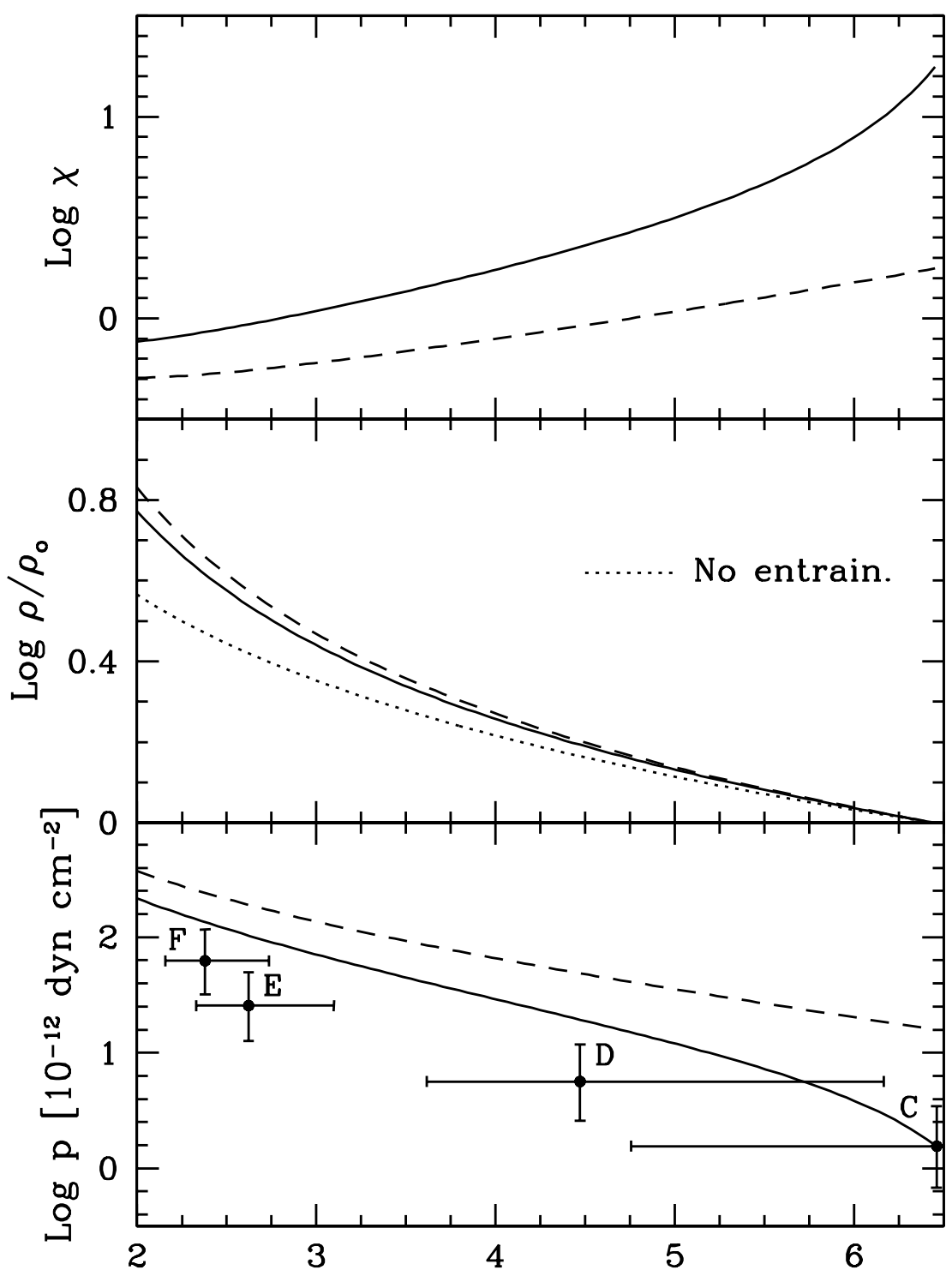

